\title{
OPTIMAL SELECTION OF THE CA-CFAR ADJUSTMENT FACTOR FOR K POWER SEA CLUTTER WITH STATISTICAL VARIATIONS
}

\section{SELECCIÓN ÓPTIMA DEL FACTOR DE AJUSTE CA-CFAR PARA CLUTTER MARINO DE POTENCIA K ESTADÍSTICAMENTE VARIABLE}

\author{
José Raúl Machado Fernández', Jesús C. Bacallao Vidal²
}

Fecha de recepción: 9 de febrero de 2016

Fecha de revisión: 7 de julio de 2016

Fecha de aprobación: 8 de agosto de 2016

Referencia: J. R. Machado Fernández, J. C. Bacallao Vidal (2017). Optimal selection of the CA-CFAR adjustment factor for K power sea clutter with statistical variations. Ciencia e Ingeniería Neogranadina, 27 (1), pp. 61-76, DOI: http://dx.doi. org/10.18359/rcin. 1714

\begin{abstract}
The presence of the sea clutter interfering signal sets limitations on the quality of radar detection in coastal and ocean environments. The CA-CFAR processor is the classic solution for detecting radar targets. It usually operates keeping constant its adjustment factor during the entire operation period. As a consequence, the scheme does not take into account the slow statistical variations of the background signal when performing the clutter discrimination. To solve this problem, the authors conducted an intensive processing of 40 million computergenerated clutter power samples in MATLAB. As a result, they found the optimal adjustment factor values to be applied in 40 possible clutter statistical states, suggesting thus the use of the CA-CFAR architecture with a variable adjustment factor. In addition, a curve fitting procedure was performed, obtaining mathematical expressions that generalize the results for the whole

1. Ingeniero en Telecomunicaciones y Electrónica, Doctorante, Profesor e Investigador, Grupo de Investigación de Radares, Departamento de Telecomunicaciones y Telemática, Facultad de Eléctrica, Universidad Tecnológica de La Habana José Antonio Echeverría (CUJAE), La Habana, Cuba, josemf@electrica.cujae.edu.cu

2. Ingeniero Eléctrico, Doctor en Ciencias Técnicas, Profesor Titular e Investigador, 2do Jefe del Grupo de Investigación de Radares, Departamento de Telecomunicaciones y Telemática, Facultad de Eléctrica, Universidad Tecnológica de La Habana José Antonio Echeverría (CUJAE), La Habana, Cuba, bacallao@electrica.cujae.edu.cu
\end{abstract}


addressed range of clutter statistical states. The experiments were executed with a 64 cells CA-CFAR and found the adjustment factor values for three common false alarms probabilities. The $\mathrm{K}$ distribution was used as clutter model, thanks to its wide popularity. This paper facilitates the handling of the $\mathrm{K}$ power distribution avoiding the use of Gamma and Bessel functions, commonly found in developments related to the $\mathrm{K}$ model. Moreover, requirements for building an adaptive clutter detector in $\mathrm{K}$ power clutter with a priori knowledge of the shape parameter were fulfill. Also, several recommendations are given to continue the development of a more overall solution which will also include the estimation of the shape parameter.

Keywords: sea clutter, $\mathrm{K}$ power distribution, CA-CFAR detector, selection of the adjustment factor, adaptive detection thresholds.

\section{RESUMEN}

La presencia de la señal interferente de clutter marino establece limitaciones en la calidad de la detección de radar en ambientes costeros y de alta mar. El procesador CA-CFAR es la solución clásica para detectar blancos de radar. Usualmente mantiene su factor de ajuste constante todo el período de operación. Como consecuencia, el esquema no toma en consideración las variaciones estadísticas de la señal de fondo cuando realiza la discriminación del clutter. Para resolver este problema, los autores realizaron un procesamiento intensivo de 40 millones de muestras de clutter de intensidad, generadas en computadora a través de MATLAB. Como resultado, encontraron los valores óptimos del factor de ajuste a ser aplicados para 40 posibles estados estadísticos del clutter, sugiriendo el uso de la arquitectura CA-CFAR con un factor de ajuste variable. Adicionalmente, fue llevado a cabo un ajuste de curvas, obteniéndose expresiones matemáticas que generalizan los resultados en todo el intervalo de considerado de estados estadísticos del clutter. Los experimentos se ejecutaron con un CA-CFAR de 64 celdas y apuntaron a encontrar los valores del factor de ajuste para tres probabilidades de falsa alarma comunes. La distribución K fue elegida como el modelo usado para el clutter, gracias a su amplia popularidad. Este artículo facilita el manejo de la distribución $\mathrm{K}$ de intensidad, evitando el uso de funciones Gamma y Bessel, comúnmente encontradas en desarrollos relacionados con el modelo K. Además, fueron cumplidos los requerimientos necesarios para construir un detector adaptativo en clutter de potencia $\mathrm{K}$ con conocimiento previo del parámetro de forma. Al mismo tiempo, fueron dadas varias recomendaciones para continuar el desarrollo de una solución más general que también incluirá la estimación del parámetro de forma.

Palabras clave: clutter marino, distribución $\mathrm{K}$ de la potencia, detector de promediación CA-CFAR, selección del factor de ajuste, adaptación del umbral de detección. 


\section{INTRODUCTION}

The purpose of a radar device is to detect nearby objects within a certain action range. The device sends electromagnetic signals in different directions hoping that some of them will impact on a target. The echo signal produced on the target surface is also dispersed in multiple directions and only a small fraction returns back to the radar antenna. With the information contained in the echo signal, modern radars are able to estimate several target parameters such as speed and position [1].

However, in a real scenario, in addition to the target echo, the radar receives another contribution produced in the area surrounding the target. This random input is known as clutter [2].

Due to the presence of clutter and receiver thermal noise, radars are unable to ensure a completely reliable detection. Actually, one of the fundamental parameters of a radar is the false alarm probability $(P)$ that quantifies the amount of mistakes made by the device when it classifies clutter samples as targets. Radar processors seek to guarantee the CFAR (Constant False Alarm Rate) property and to operate with a constant false alarm probability $[3,4]$.

There are several types of CFAR algorithms, all based on the sliding window mechanism [5]. The most popular are the CA-CFAR (Cell Averaging-CFAR), the GO-CFAR (Greatest-Of CFAR), the SO-CFAR (Smaller-of CFAR) and the OS-CFAR (Ordered Statistics-CFAR). These detectors have been treated in the literature by different authors $[4,6,7]$ and are frequently used as a comparative reference on current researches [8-11]. Additionally, new alternatives are presented each year in various international journals. Some proposals seek to introduce new processing methods $[11,12]$; while others focus on improving existing ones $[13,14]$.

Despite the diversity of CFAR alternatives, most implementations have in common that they allow the adjustment of the false alarm probability $\left(P_{f}\right)$ by means of the modification of a scale or adjustment factor $(T)$. By increasing, the designer ensures a better value of $P_{f}$ but the probability of detection $\left(P_{d}\right)$ drops proportionally to the $P_{f}$ gain $[4,6]$. For solving this issue, the Neyman-Pearson criterion is applied in radar design. It states that the $P_{f}$ requirement should be met at first, and only then the improvement of the $P_{d}$ can be considered by modifying other parameters.

\section{Motivation and objectives}

As it was explained on [4], CFAR detectors, and more specifically the CA-CFAR, usually establish a fixed $T$ through the whole operation time, which guarantees a constant $P_{f}$ in homogeneous clutter. However, in [15] it was proved that the CA-CFAR scheme is unable to maintain the design $P_{f}$ when facing homogeneous clutter with a variable statistic. Moreover, this result can be generalized for most CFAR processors.

It was also stated in [15] that, in order to maintain a true constant false alarm probability, a correction of the adjustment factor (T) was necessary in response to the clutter statistical variation. Statistical changes that occur in the clutter have been described in many publications [16-18]. These changes are mathematically represented as a variation of the shape parameter of the model assumed for the clutter. 
At present, the Havana Technological University (CUJAE) radar research team concentrates its efforts on the design of detectors adapted to slow clutter statistical changes. The popular Weibull $[19,20]$ and Log-Normal $[21,22]$ distributions, often used for land and sea clutter representation, have been modeled in MATLAB and addressed in adaptive solutions given in [23-25].

Nevertheless, in the specific case of the $K$ distribution [26, 27], widely regarded as the best sea clutter model, solutions have not been yet developed due to the complexity of the distribution's definition that includes a Bessel and a Gamma functions, as well as separate versions for amplitude and power signals. However, given the recent modeling of the $\mathrm{K}$ distribution in MATLAB achieved by the CUJAE research group, the authors have now the mathematical and computer fundamentals necessary to build adaptive solutions under $\mathrm{K}$ clutter.

Building an adaptive solution will first require a study on the selection of the optimal values of $T$ to be used in the range of possible clutter states. Clutter states are defined, in the bi-parametric $\mathrm{K}$ power distribution, through the shape parameter often denoted as $v$. After consulting several papers, the authors found that the possible $v$ values go from 0,1 to $30[16,28,29]$. The second requirement for building an adaptive solution is to estimate $v$ from a finite amount of clutter samples.

The objective of the current investigation was to solve the first of the two previous requirements. For that purpose, the authors conducted experiments with 40 million computergenerated $\mathrm{K}$ samples to find the optimal $T$ for $40 v$ figures in the range from 0,1 to 30 . Then, a curve fitting procedure was executed with the found $T$ values in order to generalize the results for the whole $v$ range. As a result, mathematical expressions were found providing an optimal selection of $T$ for $K$ power distributed sea clutter with statistical variations.

The paper proceeds as follows. The next section, under the name of "Materials and Methods" provides the mathematical foundations of the $\mathrm{K}$ power distribution. In the same section, some simulation variables are discussed and the conducted experiment is described. Then, in "Results" the contribution of the research is presented by offering $120 \mathrm{~T}$ values to be applied when operating with a CA-CFAR against $K$ power clutter with 40 different $v$ figures. These values are then summarized into three mathematical expressions that allow the generalization of results. The "Discussion" section comes next, performing a further analysis on the results, presenting several comparisons and extracting recommendations from the study. Finally, "Conclusions and Future Work" provides the key points of the paper and describes the future research lines for the CUJAE radar research group.

\section{MATERIALS AND METHODS}

The "Materials and Methods" section is divided into three sub-sections. In the first one, the mathematical foundations of the $\mathrm{K}$ power distribution are presented; whereas the second one discusses some simulation variables. The third sub-section describes the experiment. The aim of the section is to facilitate the reproduction of the study.

\section{$1.1 \mathrm{~K}$ power distribution}

In many radar applications, the intensity of the $K$ distribution envelope is used as clutter model 
because the detection is made through the square law detector. The $\mathrm{K}$ power distribution can be obtained by the multiplication of two components: the square of the local mean and the square of the speckle, as it is shown below [30]:

$$
|\widetilde{X}|^{2}=Y^{2} \times|\widetilde{S}|^{2} \quad W=Z \times R
$$

The local average, $Y$, is Root-Gamma distributed; while $Z$ is Gamma distributed:

$$
f_{Z}(z)=\frac{b^{2 v}}{\Gamma(v)} z^{v-1} \exp \left(-b^{2} z\right)
$$

The amplitude of the speckle component, $\tilde{\mathrm{S}}$, is Rayleigh distributed and its squared version, $R$, has an exponential PDF:

$$
f_{R}(r)=\frac{1}{2 \sigma^{2}} \exp \left(-\frac{r}{2 \sigma^{2}}\right)
$$
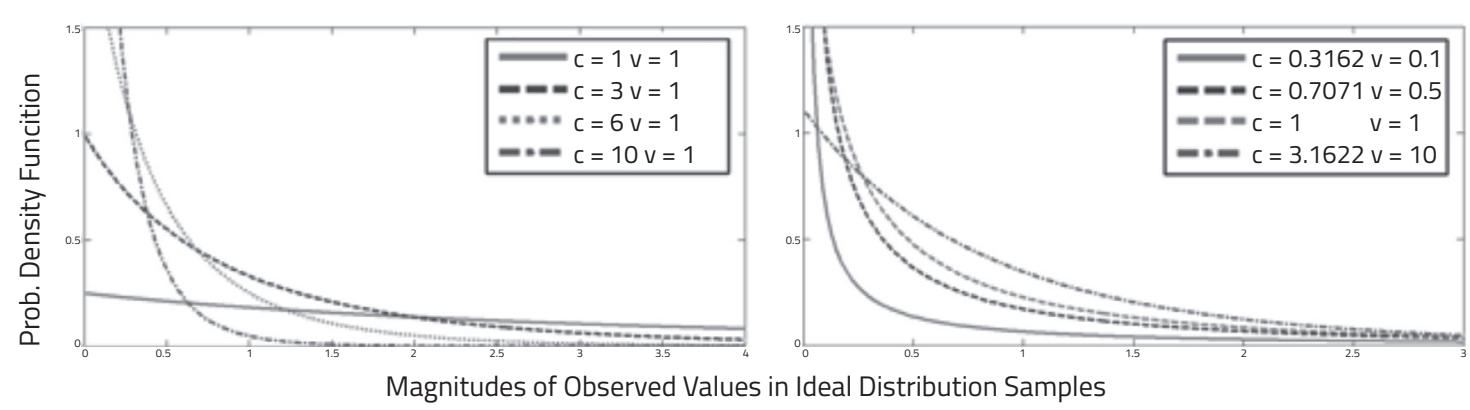

Magnitudes of Observed Values in Ideal Distribution Samples

The PDF of the $\mathrm{K}$ power clutter distribution is [30]:

$f_{W}(w)=\frac{2 c^{v+1}}{\Gamma(v)} w^{\frac{v-1}{2}} K_{v-1}(2 c \sqrt{w})$ with $c=\sqrt{\frac{\pi}{2}} b$

Where $b$ and $c$ are the scale parameters and $v$ is the shape parameter; $K($.$) is the modified$ Bessel function of second kind and $\Gamma($.$) is the$ Gamma function. In Figure 1, the influence of the parameters in the $\mathrm{K}$ intensity PDF curve can be appreciated.

Finally, the algebraic moments of order of the K power distribution are:

$$
E\left\langle w^{n}\right\rangle=\frac{1}{c^{2 n}} \frac{\Gamma(v+n)}{\Gamma(v)} \Gamma(n+1)
$$

\subsection{Selection of simulation variables}

To achieve the aimed objective, the authors had to make three fundamental decisions regarding

Fig. 1. Effect of the variation of the parameters on the shape of the $K$ power PDF.

Source: The authors. 
the variables included in the simulations. Firstly, it was necessary to choose the size of the CA-CFAR sliding window. This window moves all around the coverage area, so that each cell has a chance to occupy the window's central cell in a given moment of time. The central cell is always the one under evaluation for deciding on the presence of a target. The CA-CFAR mechanism estimates the clutter mean magnitude by averaging the values from the non-central cells. Therefore, higher window sizes achieve a high precision in the estimated average while smaller sizes are associated with lower processing loads. Fortunately, the existence of previous similar studies facilitated the decision. As in [24, 25], a window size of 64 cells, which provides a good balance between gain and resource consumption, was chosen.

Secondly, the authors needed to choose the number of samples of the sets that would be included in the experiments. A very small amount of samples will produce a poor estimate of the resulting optimal $T$; while processing too many samples may significantly slow down the execution of the experiments. In brief, it was decided that one million samples was high enough to ensure a good accuracy in the results without incurring in a too high processing time. This value is closely related to the $P_{f}$ wanted figures, which are described below.

Finally, it was necessary to decide which $P_{f}$ values should be addressed in the trials. Note that as the searched $P_{f}$ value gets smaller it will become necessary to choose sets with more samples. Also by consulting the procedure followed in $[24,25]$, the authors selected the false alarm probability values of: $P_{f}=10^{-2}, P_{f}=10^{-3}$ and $P_{f}=10^{-4}$.

It's important to understand that the increase of $T$ will always benefit the $P_{f}$ but it will also cause a drop in the probability of detection; therefore choosing a too high $T$ value is not a viable option. Precisely, the purpose of the current study is to find the exact $T$ value for which the wanted $P_{f}$ s are found with no extra loss on the $P_{d}$.

Figure 2 illustrates the effect of $T$ modification on the detection threshold. A too low $T$ value will produce a very good $P_{d}$ because it will cause the threshold to stay low which will result in the detection of most targets; however, this low threshold will also cause high magnitude clutter samples to be classified as targets, resulting in a very poor $P_{f}$ figure. The opposite effect is obtained when a very high $T$ is selected.

\subsection{Algorithm for the estimation of the optimal factors}

After discussing the selection of the variables included in the simulation, the current subsection explains the structure of the generated samples set and describes the algorithm that produced the results.

The employed set contained 40 groups of one million $\mathrm{K}$ power samples each. Each group was computer-generated by using MATLAB 2011 and a different $v$ value. The $v$ values covered the range between 0,095 and 31, slightly exceeding the range found in literature from 0,01 to $30[16,28,29]$. More specifically, several $v$ values between 2 and 25 were found in the study of [29]. The lower limit was extended to 0,1 in [16], whereas the upper limit was expanded to 30 in [28].

The 0,095-31 range was not covered with a uniform sweep, but by selecting more values in the interval from 0,01 to 1 . This was done 


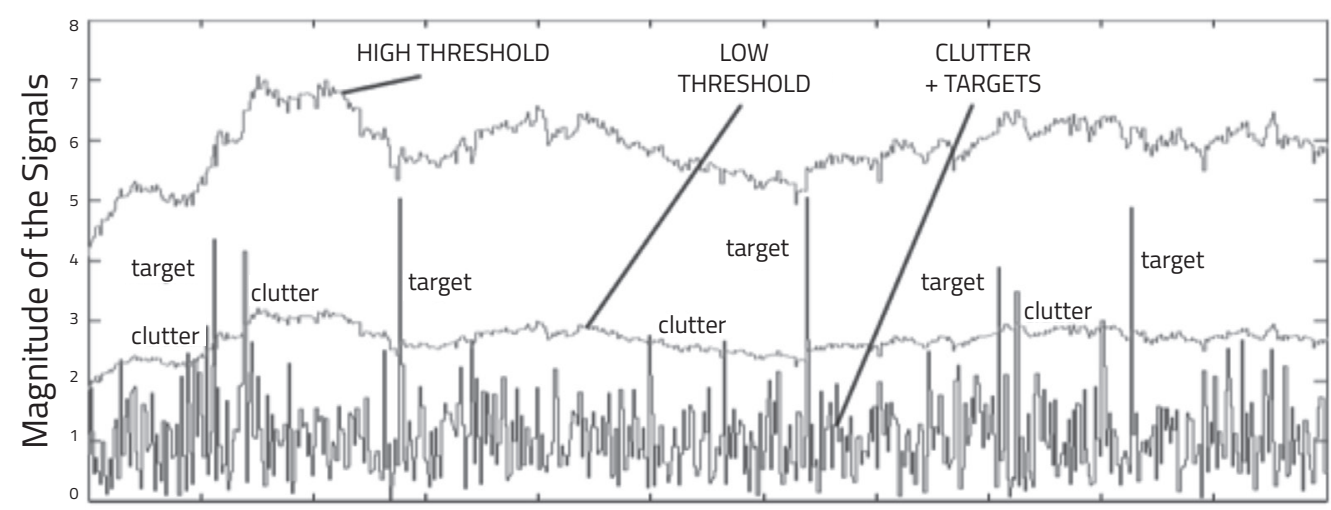

Sequence of Clutter + Target Samples

Fig. 2. Consequence of modification on the detection threshold.

Source: The authors.

because it was detected in preliminary experiments that figures between 0,01 and 1 had a marked influence on the heavy property of the $\mathrm{K}$ power distribution tails. More specifically, the first selected $v$ value was 0,095 and the next one was a $16 \%$ higher. This rule was maintained until the 40th value was obtained. For clarity, employed $v$ values are shown in Table 1, ordered from left to right and from top to bottom. Meanwhile, the $\mathrm{K}$ scale parameter remained as $c=\sqrt{v}$ to fix the mean of the samples to one, as suggested in [30].
The executed algorithm will be described next. Initially, the first group of one million samples was selected and processed with a CA-CFAR operating with a small adjustment factor such as $T=1,1$. Then, the $P_{f}$ was measured using the following expression:

$$
P f=\frac{\text { false positives }}{\text { total amount of samples }}
$$

where false positives are clutter samples mistakenly identified as targets by the CA-CFAR

Table 1. Values of $v$ used in the experiment.

\begin{tabular}{|c|c|c|c|c|c|c|c|c|c|}
\hline 0,095 & 0,1102 & 0,1278 & 0,1482 & 0,172 & 0,1995 & 0,2314 & 0,2684 & 0,3114 & 0,3612 \\
\hline 0,419 & 0,4861 & 0,5639 & 0,6541 & 0,7588 & 0,8802 & 1,021 & 1,1844 & 1,3739 & 1,5937 \\
\hline 1,8487 & 2,1445 & 2,4877 & 2,8857 & 3,3474 & 3,883 & 4,5043 & 5,225 & 6,061 & 7,0308 \\
\hline 8,1557 & 9,4606 & 10,9743 & 12,7302 & 14,7671 & 17,1298 & 19,8706 & 23,0499 & 26,7378 & 31,0159 \\
\hline
\end{tabular}

Source: The authors. 
mechanism. Note that the simulations were performed with no targets present, so all positive detections were false positives.

As the initial $T$ was very low, a high $P_{f}$ was obtained, such as $P_{f}=0,009$. The next iteration slightly increased $T$ and the $P_{f}$ was measured again. This process was repeated until the optimal $T$ values were found achieving less than a $1 \%$ deviation from the three addressed $P_{f}\left(P_{f}=10^{-2}, P_{f}=10^{-3}\right.$ and $\left.P_{f}=10^{-4}\right)$. At this point, trails were completed for the first group of one million samples. The procedure was repeated on the remaining groups and ended when the three $P_{f}$ were found for all groups, resulting in $120 T$ values. A summary of the implemented algorithm is given below:

\section{Repeat 40 times \\ In each repetition: Select one pair \\ Repeat until the wanted $P_{f}$ are found (around 40 repetitions) \\ In each repetition: Increase $T$ looking for the wanted $P_{f}$ \\ End of Repetitions \\ End of Repetitions}

The results drawn from this experiment are said to be optimal because the previously described algorithm is in fact a binary search that minimizes the error to $1 \%$. The same algorithm was executed in [24] for the Weibull distribution resulting in 18 values for the shape parameter.

\section{RESULTS}

By executing the previously specified algorithm, $120 T$ values were found and plotted on Figure 3. As it's clearly visible, data belonging to the three false alarm probabilities follow a common trend: high $T$ values are obtained for low $v$ figures and low $T$ values for high. Also, the influence of on the modification of fades with the increase of the shape parameter.

In order to synthesize the found values in easily applicable mathematical expressions, different types of curve fittings were performed. Figure 4 shows the lack of proximity between the data and polynomial fits of the first three orders, a trend that continued until the eighth order.

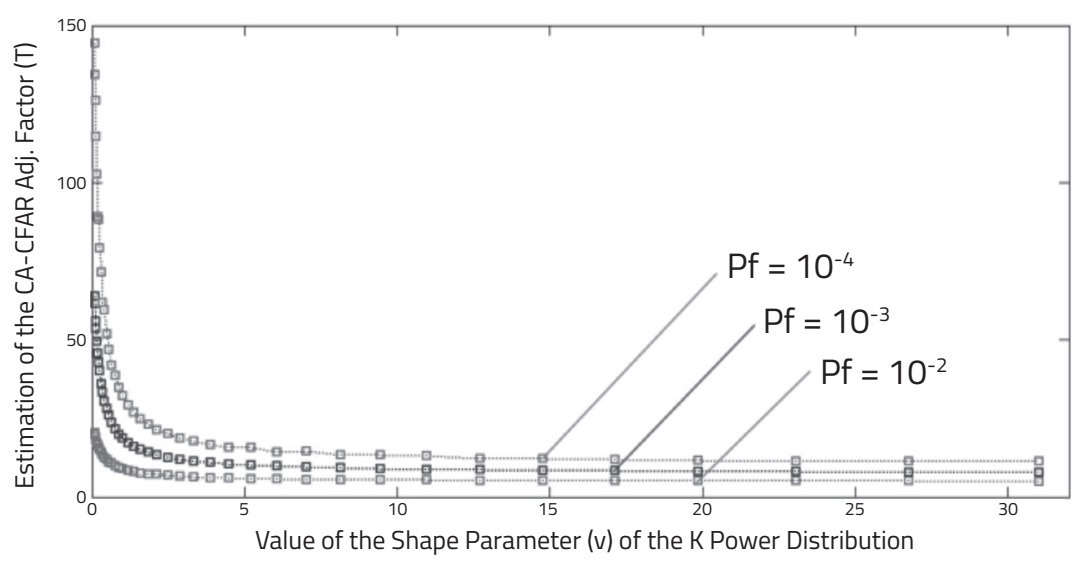

Fig. 3. Estimated values for the $40 T$ addressed $v$.

Source: The authors. 

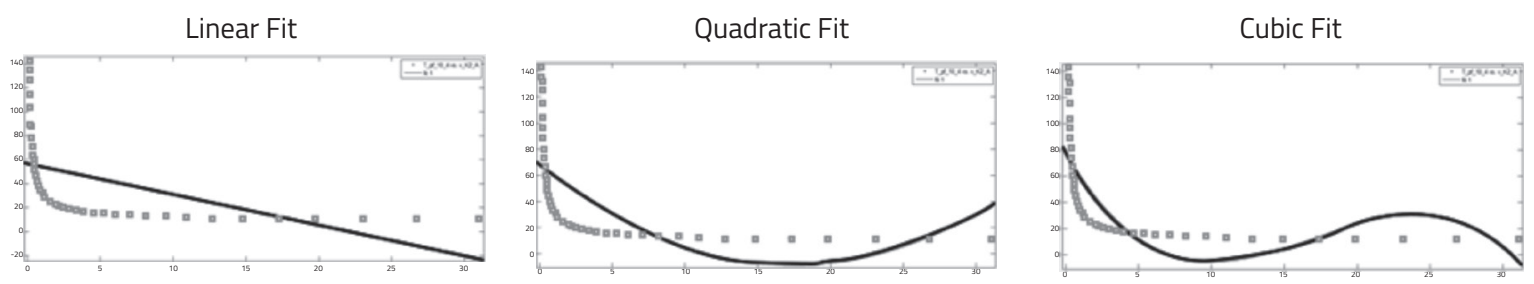

Fig. 4. Linear Fit of the first three orders for $T$ values from $P_{f}=10^{-4}$.

Source: The authors.

Finally, after trying several alternatives, the power fit showed a good closeness to the data. Figure 5 shows the goodness of fit achieved for $P_{f}=10^{-4}$ in graph number one. A similar behavior was found for $P_{f}=10^{-3}$ and $P_{f}=10^{-2}$.

The authors noted that even if the fit was globally good, deviations occurred for $v \geq 10$ values, where the fit line was always inferior to the data. The probable cause is the high magnitude and the large quantity of $v \leq 1$ values considered in the fit. If an error is to be permitted in the fit, it should be an excess error that would suggest using $T$ values higher than the optimal ones. The behavior displayed by graph 1 from Figure 5 for $v \geq 10$ values will cause the detector to operate with a $T$ lower than the optimal, which will go in detriment of the $P_{f}$ Therefore, the detector will no longer comply with the Neyman-Pearson theorem.

In order to correct the problem and to improve the fit, it was decided to divide the $v$ range into two intervals. After testing various possibilities, it was found that the value of $v=3,5$ could be used as a boundary. The two new power fits are shown in graphs 2 and 3 from Figure 5. The first covers the $0 \leq v \leq 3,5$ interval and the second $3,5 \leq v \leq 30$ interval. It can be observed in graph 3 , that the fit solves the previously encountered problem by displaying positives and negatives mistakes for $v \geq 10$ and avoiding the marked decreasing tendency.

Table 2 shows the results of the fit made for the whole $v$ interval. The well-know RMSE (Root Mean Squared Error) and SSE (Sum of Squared Error) are provided together with valid expressions for each $P_{f}$ Tables 3 and 4 do the same for the $0 \leq v \leq 3,5$ and $3,5 \leq v \leq 30$ fits.

\section{DISCUSSION}

The main contribution of the paper is to provide the mathematical expressions required for the optimal selection of $T$ in $\mathrm{K}$ power sea clutter with statistical variations. These expressions allow the building of an adaptive detector in $K$ power clutter with the a priori knowledge of the shape parameter.

In addition, the paper also encourages the creation of a more overall solution which will also include the shape parameter estimation. Investigations similar to this one were carried out in [24] for Weibull clutter, leading to the subsequent design of an overall adaptive scheme in [25]. Expressions found here fulfill half of the requirements necessary to design CA-CFAR improved detectors which will replace the contribution of [25] in sea environ- 


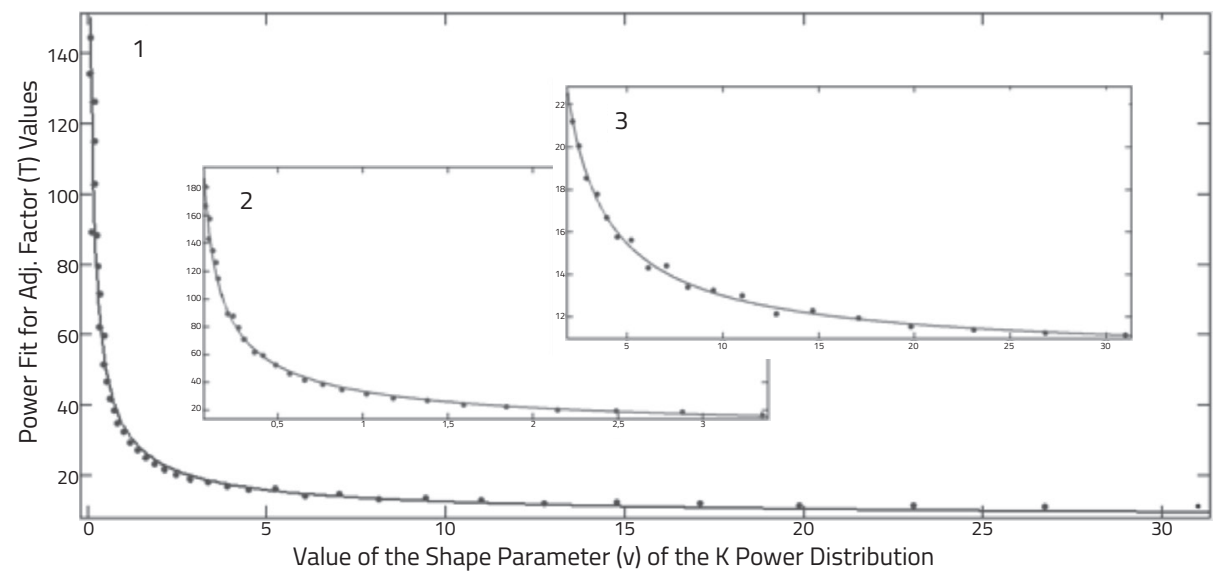

Fig. 5. Power fit for data belonging to $P_{f}=10^{-4}$.

Source: The authors.

Table 2. Power fit numerical results for the $\boldsymbol{v}$ whole range.

\begin{tabular}{|c|c|c|c|}
\hline False Alarm Prob. & Expressions found through the fit & RMSE & SSE \\
\hline$P_{f}=10^{-2}$ & $f(x)=5,876 x^{-0.4765}+3,354$ & 0,3867 & 5,532 \\
\hline$P_{f}=10^{-3}$ & $f(x)=14,77 x^{-0.6094}+4,974$ & 1,036 & 39,73 \\
\hline$P_{f}=10^{-4}$ & $f(x)=27,15 x^{-0.7058}+7,022$ & 2,09 & 161,6 \\
\hline
\end{tabular}

Source: The authors.

Tabla 3. Power fit numerical results for $0 \leq v \leq 3,5$ the interval.

\begin{tabular}{|c|c|c|c|}
\hline False Alarm Prob. & Expressions found through the fit & RMSE & SSE \\
\hline$P_{f}=10^{-2}$ & $f(x)=10,28 x^{-0.3223}-1,048$ & 0,2669 & 1,567 \\
\hline$P_{f}=10^{-3}$ & $f(x)=20,36 x^{-0.5037}-0,8328$ & 0,8626 & 16,37 \\
\hline$P_{f}=10^{-4}$ & $f(x)=33,54 x^{-0.6309}+0,06963$ & 2,108 & 97,8 \\
\hline
\end{tabular}

Source: The authors.

Tabla 4. Power fit Numerical results for the $3,5 \leq v \leq 30$ interval.

\begin{tabular}{|c|c|c|c|}
\hline False Alarm Prob. & Expressions found through the fit & RMSE & SSE \\
\hline$P_{f}=10^{-2}$ & $f(x)=4,436 x^{-0,8515}+4,739$ & 0,02026 & 0,006565 \\
\hline$P_{f}=10^{-3}$ & $f(x)=11,49 x^{-0,8331}+7,203$ & 0,04251 & 0,02891 \\
\hline$P_{f}=10^{-4}$ & $f(x)=21,66 x^{-0.8532}+9,957$ & 0,2206 & 0,7784 \\
\hline
\end{tabular}

Source: The authors. 
ments, where the $\mathrm{K}$ model has been proven superior.

The CUJAE radar research group plans to use the achieved results in the design of two types of detectors. Firstly, the authors will apply the method of moments in the estimation of the $v$ parameter for designing the K-MoM-CA-CFAR (K Method of Moments CA-CFAR). Secondly, the construction of the K-NATE-CA-CFAR (K Neural Adaptive Threshold Estimation (A-CFAR) will be addressed, developing a system similar to the one presented in [25]. Additionally, the method developed here for optimal $T$ selection will be included in the MATE-CFAR 2 (MAtlab Test Environment for CFAR detectors 2) library which is a progression of MATE-CFAR Version 1 presented in [15], as an answer to the need of creating threshold adaptive mechanism pointed out in [3].

The algorithm used by the authors for obtaining the optimal $T$, which is based on Monte Carlo simulations, it's appropriate for the $\mathrm{K}$ distribution given its intricate mathematical definition. Comparable executions can be found in [31].

In addition, according to the procedure followed in [24], it's useful to analyze the derivative of the optimal $T$ values, that is, the difference between each value and the next one. Nevertheless, the authors of this paper chose to offer a more concise presentation of the derivatives than the one provided in [24]. To do so, they joined the derived contributions from each of the three treated $P_{f}$ in the graph from Figure 6.

The performed procedure was as follows. First, the derivative was calculated for each of the three sets of $T$ values plotted in Figure 3. Then, derivatives were divided by the absolute quantities from Figure 3 yielding a relative derivative. Finally, the three relative derivatives were averaged and multiplied by 100 , producing the percentage of the relative derivative average shown in Figure 6.

Thanks to Figure 6, the influence of $v$ increase on the selection of the optimal $T$ values can

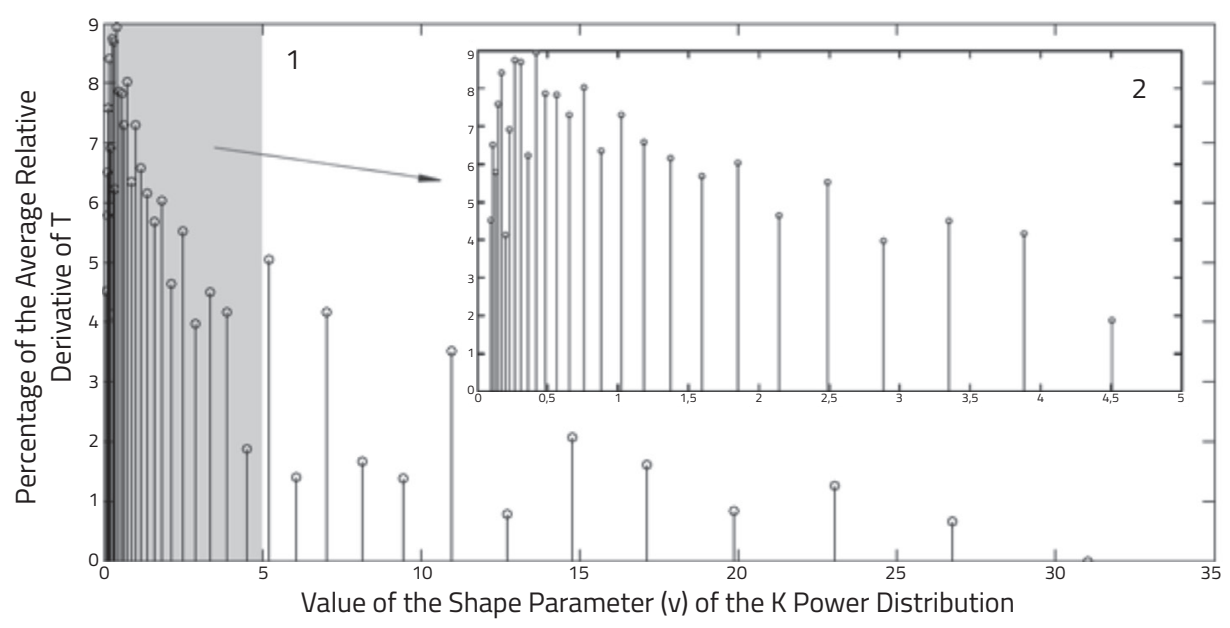

Fig. 6. Percentage of the $T$ relative derivative average for the three addressed $P_{f}$

Source: The authors. 
be clearly appreciated for the whole three $P_{f}$ Notice how for high values of $v$ the percentage of the derivative is smaller even though the distance between consecutive values is far superior to that exhibited for small figures of $v$.

Precisely, according to the non-uniform sampling performed on the $\mathrm{K}$ distribution's $v$ values, there are far more tests related to lower $v$ values than tests related to high values. Therefore, the visibility is reduced for $v<1$ in Graph 1 from Figure 6. For solving this issue, Graph 2 was added, also inside Figure 6, for reproducing the behavior of the shaded region from Figure 1. Thus an improved visualization of the percentage of the derivatives is achieved.

Using the data from Figure 6 and performing some auxiliary calculations, the authors were able to divide the behavior of $T$ variation in four key intervals which are presented in Table 5. The first two columns of the table are easy to understand: they indicate (1) the number associated to each interval and (2) the limits of the contained $v$ values (abscissa axis from Figure 6).

The third column from Table 5 provides the number of sweeps in the interval, that is, the number of $v$ values contained. Moreover, the fourth column displays the average of the derivative calculated from one sweep to another within the same interval.

The fifth and sixth columns provide global information of the derivative of $T$ regarding $v$ values inside the interval. The fifth column shows the derivative between the edges, that is, the change that occurred between the first value and the last one from each interval. Column number six tabulates the change experienced per $v$ unit.

Table 5 confirms the relationship observed in Figure 6 regarding the reduced influence of high $v$ values on the selection of the optimal $T$. For example, the modification of $v$ within Interval 4 has a reduced influence on $T$ selection. Observe that, despite the large extension of the fourth interval $(10<v<30)$ and the small amount of sweeps contained on it, the average derivative between consecutives sweeps is only of $0,728 \%$, the derivative between the boundaries is only of $4,98 \%$ and the change produced on $T$ per $v$ unit (for examples between $v=14$ and $v=15$ ) is only of $0,25 \%$. This is in high contrast with the behavior observed for Intervals 1 and 2, especially in Interval 1 where a slight variation of $v$ results in a remarkable modification of $T$ the optimal value.

Table 5. Numerical data extracted from Figure 6.

\begin{tabular}{|c|c|c|c|c|c|}
\hline $\begin{array}{c}\text { Interval } \\
\text { Number }\end{array}$ & Interval Boundary & $\begin{array}{c}\text { Amount of } \\
\text { Sweeps }\end{array}$ & $\begin{array}{c}\text { Derivative } \\
\text { Average }\end{array}$ & $\begin{array}{c}\text { Derivative between } \\
\text { Edges }\end{array}$ & $\begin{array}{c}\text { Derivative per } \\
\text { unit }\end{array}$ \\
\hline Interval 1 & $0,095 \leq v<1$ & 17 & $7,12 \%$ & $70 \%$ & $77,3 \%$ \\
\hline Interval 2 & $1 \leq v<3,5$ & 7 & $5,39 \%$ & $32,6 \%$ & $13 \%$ \\
\hline Inverval 3 & $3,5 \leq v<10$ & 7 & $2,9 \%$ & $18,2 \%$ & $2,8 \%$ \\
\hline Interval 4 & $10 \leq v<30$ & 6 & $1,0305 \%$ & $7 \%$ & $0.35 \%$ \\
\hline
\end{tabular}

Source: The authors. 
The division made on Table 5 constitutes a recommendation on the separation of the training set for the design of neural $v$ estimators, as the one constructed in [25]. This type of estimators tends to keep their performance constant through the whole estimation interval. This property is commonly regarded as beneficial but it could prove to act against the overall result if it's used in the estimation of $v$, whose influence on the selection of $T$ decreases remarkably as $v$ gets higher. According to the results shown in Table 5, it's necessary to obtain a much higher precision in the estimation of the $\mathrm{K}$ shape parameter in the region between $0 \leq v \leq 1$.

As it was previously done in the solution developed in [32], a good method for training a neural network for the $\mathrm{K}$ shape parameter is to divide the estimation region into several portions. Another alternative is to oversample the interval with a greater influence on $T$ (in this case $0 \leq v<1$ ), indicating to the network that it should pay more attention to the data contained on it.

Data from Table 5 also helps understanding the nature of $\mathrm{K}$ clutter modeling. The region from $0 \leq v<1$ corresponds to great changes on the $T$ selection, which indicates that it represents a very spiky clutter (one in which high magnitude samples occur with frequency). The three following regions display, in a successive way, a reduction on the $T$ variation and represent thus less spiky clutter situations. It's important to notice that the adjustment factor is an excellent measure of the heavy property of the K PDF.

\section{CONCLUSIONS AND FUTURE WORK}

Expressions that provide the optimal values of the adjustment factor were found for a
64 cells CA-CFAR facing statistically variable clutter. The statistical variation was simulated by assuming a clutter distributed by a $\mathrm{K}$ power law with a variable shape parameter in the range from 0,01 to 30 . The results allow the creation of an improved CA-CFAR detector that will keep a truly constant false alarm rate by correcting its adjustment factor in response to a priori know clutter variations. In addition, a more overall solution, that will include the estimation of the shape parameter, is suggested and several recommendations are given for its construction. The found expressions are valid for the false alarm probabilities of 0,01, 0,001 and 0,0001 and were obtained by performing Monte Carlo simulations on 40 million computer-generated samples.

The authors plan to reproduce the investigation for Pareto clutter. Therefore, it's necessary to perform a review on the possible values of the Pareto shape parameter and to model the Pareto distribution in MATLAB. Likewise, as it was suggested in the paper, the authors will focus next on the creation of overall adaptive solutions on $\mathrm{K}$ power clutter, which will include the estimation of the shape parameter by applying the method of moments and neural networks.

\section{ACKNOWLEDGMENT}

The authors thank the help offered by PhD Nelson Chávez Ferry regarding the theoretical radar fundamentals and the general conception of the project. We also thank the Havana Technological University (CUJAE) which provided technical support for the development of the experiments. 


\section{REFERENCES}

[1] Melvin, W. L. \& Scheer, J. A (2014). Principles of Modern Radar, Vol III Radar Applications. New Jersey, United States of America: Scitech Publishing, pp. 1-15.

[2] Skolnik, M. I. (2008). Radar Handbook (3er edition). New York, United States of America: McGraw-Hill, pp. 15-17.

[3] Ward, K., Tough, R. \& Watts, S. (2013). Sea Clutter Scattering, the $K$ Distribution and Radar Performance (2nd edition). London, United Kingdom: The Institution of Engineering and Technology, pp. 311338. doi: 10.1049/pbra025e

[4] Rohling, H. (1983). Radar CFAR Thresholding in Clutter and Multiple Target Situations. IEEE Transactions on Aerospace and Electronic Systems, 19(4), pp. 608-621. doi: 10.1109/TAES.1983.309350

[5] Sánchez Rams, R. C. (2014). Implementación del detector CA-CFAR en VHDL para crear un PC-Radar Cubano usando FPGA (Tesis en opción al título de Ingeniero en Telecomunicaciones y Electrónica), Departamento de Telecomunicaciones y Telemática, Facultad de Ingeniería Eléctrica, Universidad Tecnológica de la Habana (CUJAE), La Habana, Cuba.

[6] Farina, A. \& Studer, F. A. (1986). A Review of CFAR Detection Techniques in Radar Systems, Microwave Journal, 29(1), pp. 115-118.

[7] Weingberg, G. V. (2004). Estimation of False Alarm Probabilities in Cell Averaging Constant False Alarm Rate Detectors via Monte Carlo Methods, Tech report ADA429631, DSTO Systems Sciences Laboratory, Salisbury, Australia.

[8] Takahashi, S. (2010). A CFAR Circuit of Detecting Spatially Correlated Target for Automotive UWB Radars (Master
Thesis), Faculty of Information Sciences, Hiroshima City University.

[9] Caso, G. \& De Nardis, L. (2013) "Cooperative Spectrum Sensing based on Majority decision under CFAR and CDR constraints". Presented in IEEE 24th International Symposium on Personal, Indoor and Mobile Radio Communications, Workshop on Cognitive Radio Medium Access Control and Network Solutions, New York, United States of America, 8-9 Sept, pp. 51 - 55. doi: 10.1109/ pimrcw.2013.6707835

[10] de Figueiredo, F. (2013). LTE Random Access Detection Based on a CA-CFAR Strategy (Thesis), Convergent Networks Department, Research and Development Center Campinas, SP - Brazil.

[11] Qin, Y. \& Gong, H. (2013). A New CFAR Detector based on Automatic Censoring Cell Averaging and Cell Averaging, TELKOMNIKA, 11(6), pp. 3298-3303. doi: 10.11591/telkomnika.v11i6.2686

[12] Van Cao, T. (2012). Non-homogeneity Detection in CFAR Reference Windows using the Mean-to-Mean Ration Test. Tech report ADA554930, DSTO Defence Science and Technology Organization, Edinburgh, Australia.

[13] Kumar Yadav, A. \& Kant, L. (2013). Moving Target Detection using VI-CFAR Algorithm on MATLAB Platform, International Journal of Advanced Research in Computer Science and Software Engineering, 3(12).

[14] Magaz, B. \& Belouchrani, A. (2011). Automatic Threshold Selection in OS-CFAR Radar Detection using Information Theoretic Criteria, Progress In Electromagnetics Research B, 30(1), pp. 157-175. doi: 10.2528/PIERB10122502

[15] Machado Fernández, J. R. \& Bacallao Vidal, J. C. (2014). MATE-CFAR: Ambiente de Pruebas para Detectores CFAR en 
MATLAB,Telem@tica,13(3),pp. 86-98.

[16] Greco, M., Bordoni, F. \& Gini, F. (2004). $X$-Band Sea-Clutter nonstationarity: Influence of Long Waves, IEEE Journal of Oceanic Engineering, 29(2), pp. 269-283.

[17] Chen, Z., Liu, X., Wu, Z. \& Wang, X. (2013). "The Analysis of Sea Clutter Statistics Characteristics Based on the Observed Sea Clutter of Ku-Band Radar". Presented in IEEE Proceedings of the International Symposium on Antennas \& Propagation, pp. 1183-1186.

[18] Watts, S. \& Rosenberg, L. (2013). "A Review of High Grazing Angle Sea Clutter". Presented at the IEEE 2013 International Conference on Radar. doi: 10.1109/RADAR.2013.6651992

[19] Fay, F. A., Clarke, J. \& Peters, R. S. (1977). "Weibull Distribution Applied to Seaclutter", Presented in Proceedings Inst. Elect. Eng. Radar Conf. '77, London, U.K., pp. 101-103.

[20] Ping, Q. (2011). "Analysis of Ocean Clutter for Wide-Band Radar Based on Real Data". Presented in Proceedings of the 2011 International Conference on Innovative Computing and Cloud Computing, Wuhan, China, pp. 121-124. doi: 10.1145/2071639.2071669

[21] Trunk, G. V. \& George, S. F. (1970). Detection of targets in Non-Gaussian Sea Clutter, IEEE Transactions, 6(1), pp. 620628. doi: 10.1109/TAES.1970.310062

[22] Ishii, S., Sayama, S. \& Mizutani, K. (2011). Effect of Changes in Sea-Surface State on Statistical Characteristics of Sea Clutter with X-band Radar, Wireless Engineering and Technology, 2(3), pp. 175-183. doi: 10.4236/wet.2011.23025

[23] Gato Martínez, I. (2014). Algoritmo para la Estimación de la Distribución del Clutter Marino (Tesis en opción al título de Ingeniero en Telecomunicaciones y
Electrónica), Departamento de Telecomunicaciones y Telemática, Facultad de Ingeniería Eléctrica, Universidad Tecnológica de la Habana (CUJAE), La Habana, Cuba.

[24] Machado Fernández, J. R. (2015). Estimation of the Relation between Weibull Distributed Sea clutter and the CA-CFAR Scale Factor, Journal of Tropical Engineering, 25(2), pp. 19-28. doi: 10.15517/jte.v25i2.18209

[25] Machado Fernández, J. R., Bacallao Vidal, J. C. \& Chávez Ferry, N. (2015). A Neural Network Approach to Weibull Distributed Sea Clutter Parameter's Estimation, Inteligencia Artificial, 18(56), pp. 3-13. doi: 10.15517/jte.v25i2. 18209

[26] Ward, K. D. (1981) Compound Representation of High Resolution Sea Clutter, Electronic Letters, 17(16), pp. 561-563. doi: 10.1049/el:19810394

[27] Abraham, D. A. \& Preston, J. R. (2010). Statistical Analysis of Monostatic and Bistatic Echoes from ShipWreck, IEEE Journal of Oceanic Engineering, 25(2), pp. 1-15.

[28] Nohara, T. J. \& Haykin, S. (1991). "Canadian East Coast Radar Trials and the K-Distribution". Presented in IEEE Proceedings on Radar and Signal Processing, pp. 80-88. doi: 10.1049/ip-f2.1991 .0013

[29] Dong, Y. (2006). Distribution of X-Band High Resolution and High Grazing Angle Sea Clutter, Technical Report DSTO-RR-0316, Electronic Warfare and Radar Division, Defence Science and Technology Organisation, Edinburgh, Australia.

[30] Cetin, A. (2008). CFAR Detection in K-Distributed Sea Clutter, Master of Science in Electrical and Electronics Engineering, The Graduate School of 
Natural and Applied Sciences of Middle East Technical University.

[31] Bocquet, S. (2012). Calculation of Radar Probability of Detection in K-Distributed Sea Clutter and Noise, Technical Report DSTO-TN-1000, Joint Operations Division, Defence Science and Technology Organization, Edinburg, Australia.

[32] Machado Gil, A. \& García Delgado, B. (2014) Reconocimiento de Parámetros Asociados a distribuciones del Clutter Marino con Redes Neuronales Artificiales (Tesis en opción Ingeniero en opción al título de Ingenieron en Telecomunicaciones y Electrónica), Grupo de Radares, Departamento de Telecomunicaciones y Telemática, IUniversidad Tecnológica de la Habana (CUJAE), La Habana, Cuba. 